\title{
Design of Solar Street Light System Based on the Maximum Power Point Tracking and Energy Output Coordination Strategy
}

\author{
Yaping $\mathrm{Wu}^{1, \mathrm{a}}$, Zekun $\mathrm{Gao}^{2, \mathrm{~b}}$ and Jie Chen ${ }^{1, \mathrm{c}}$ \\ ${ }^{1}$ Liren College, Yanshan University,Hebei Qinhuangdao, 066004, China \\ ${ }^{2}$ Computer College, Beijing Information Science and Technology University, Beijing, 100101, China \\ a yapingq@163.com, b406136159@qq.com, c63991014@qq.com
}

Keywords: Solar Street Light; Maximum power point tracking; ZigBee; CC2530; Storage battery.

\begin{abstract}
The paper presents a design of solar street lamp system based on the maximum power point tracking and energy output coordination strategy. It uses chip CC2530F256 which carries with the ADC sampling output voltage and current of the solar cell to adjust the PWM duty cycle and achieve maximum power point tracking. Besides, it uses the information of the switch of ZigBee wireless network and streetlight node battery voltage to adjust the output power of the battery, thus, achieving the reasonable control of street lighting brightness. By using a chip optimized for energy inputs and outputs, the program realized simple structure and strong practical.
\end{abstract}

\section{Introduction}

Currently, solar street light system has been widely used. How to adapt to the environment and improve the length of lighting time have become an important factor in whether it can power the street lights independently only by the solar energy. The paper presents a design based on the maximum power point tracking and energy output coordination strategy of solar lighting system. The main purpose of the maximum power point tracking is to maximize the output power of the solar array, which ensures that the battery maximize the effect of charging under the limited duration of sunshine. Output coordinated strategy is a method based on the voltage information of each node in the battery to adjust the output power of the battery. Each node is different in the external environment and internal devices also exist anisotropy so that the maximum sustainable lighting time of different street light differs in the same brightness. The system enables the exchange of information on the battery voltage for each street light via ZigBee wireless network, the coordination and control of brightness for street lights and the protection of low energy storage device, avoiding that a street light completely extinguishes due to energy depletion. The program uses CC2530 as its control center, the structure is simple and practical[1][2].

\section{Design Proposal}

The system structure of the scheme is shown as Figure 1. The system consists of solar cells, controller, batteries, solar street lamp and wireless network transmission units. Solar cells convert solar energy into electrical energy stored in the battery. The first task of the controller is to collect the output voltage and current of the solar cell. According MPPT[3], we control the size of the output current of the solar cell to achieve maximum power output of the solar cell, so that the battery can get the maximum charging efficiency. The second task of the controller is to combine the wireless unit, establish the zigbee network and exchange their battery voltage value with other nodes through the network, controlling the brightness of lights according the output coordination algorithm. The control chip of the system uses TI's CC2530F256, the chip consists of an enhanced 8051CPU and an IEEE 802.15.4-compliant radio transceiver and supports ZigBee protocol stack, with DMA controller and 8 ADC channels of 7 to 12 bit resolution. In addition, CC2530F256 has outstanding low power consumption for the usage of battery-powered system [4]. 


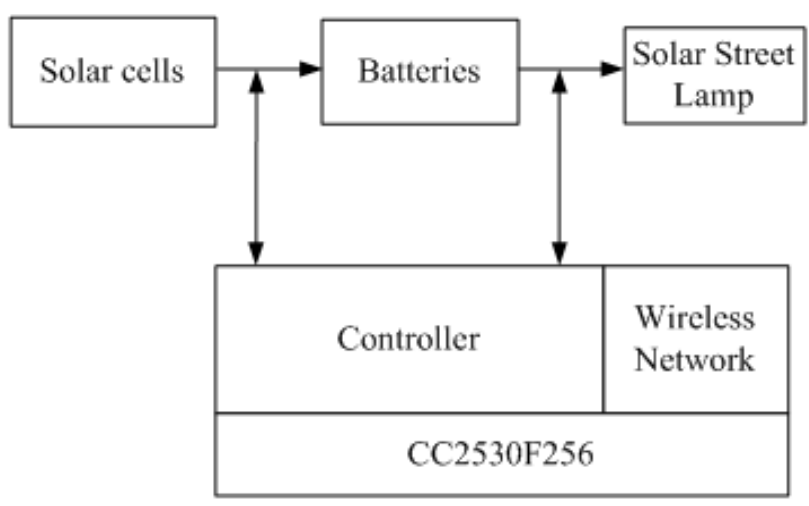

Fig.1. System Block Diagram of the Design

\section{PWM Driver Circuit Design}

In this system, there are two places using a PWM driving circuit to adjust the output current, namely, the solar cell output current regulation and battery output current regulation. Here we take the battery to brightness adjustment of lights as an example to introduce the PWM drive circuit. As shown in figure 2, CC2530 outputs PWM pulse, control MOSFET's on-off duty cycle and adjust the load current. In front of the LED (load) is smoothing circuit composed of inductor L1 and the capacitor. Even when the MOSFET is off, the diode D4 can supply power to the LED. When the PWM signal make the switch on, the current flows in accordance with the sequence of input signal switch - coil - load. Adjusting the PWM duty cycle can adjust the size of the the coil L1 (i.e. LED) current[5][6].

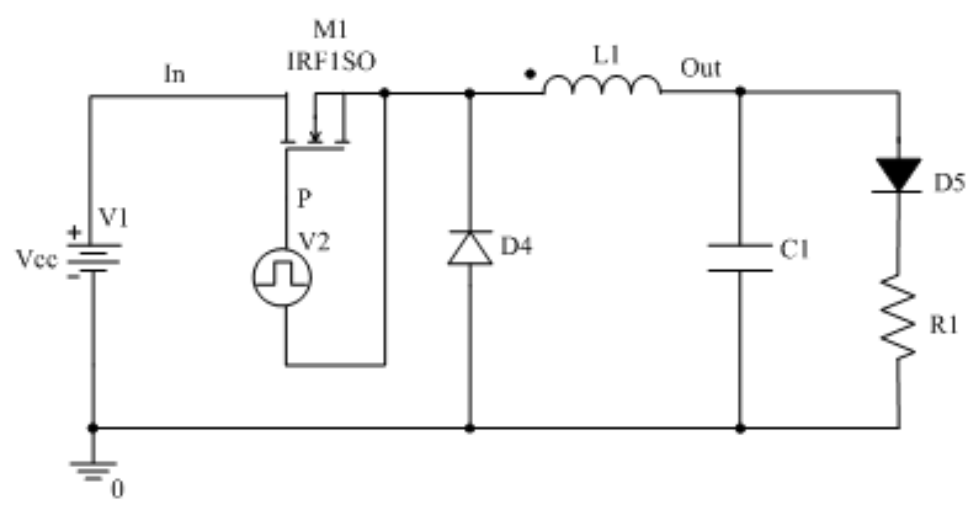

Fig.2. PWM Drive Circuit

\section{Maximum Power Point Tracking Algorithm}

In order to achieve maximum power point tracking, we use Output power comparison method here, whose basic idea is to ensure that the output power of photovoltaic cells toward the direction of the largest changes by changing the output current[7]. The system uses CC2530 comes with 12-bit ADC to measure the output voltage and current and calculate the current output power $\mathrm{P}_{\mathrm{n}}$. On the basis of present current, increase or reduce a cerain output current $\Delta \mathrm{I}$, then re-measure the output voltage and current and calculate the output power $P_{n+1}$, if $P_{n+1}>P_{n}$, just continue the direction of the disturbance before, otherwise change the direction of the disturbance. The aforementioned "increase or reduce a cerain output current $\Delta \mathrm{I}$ " is realized by the duty cycle of the PWM signal output by CC2530. Algorithm software flow chart is shown in Figure 3. 


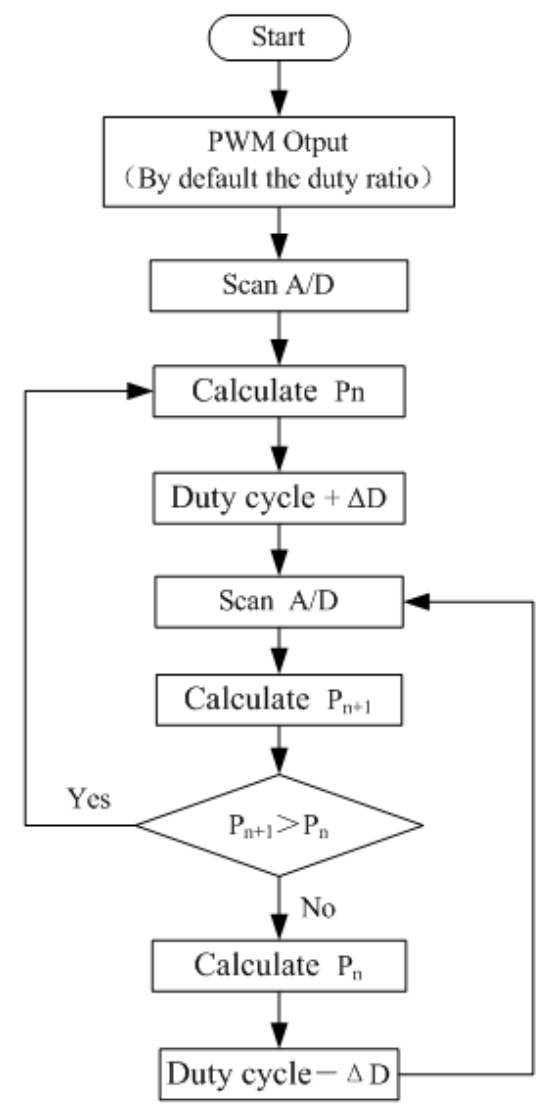

Fig.3. Flow Chart of MPPT Control

\section{Output Coordinated Strategy Algorithm}

Algorithm flow chart is shown in Figure 4. The core idea of the output power coordination strategy is that node establishes a data exchange channel through ZigBee network.

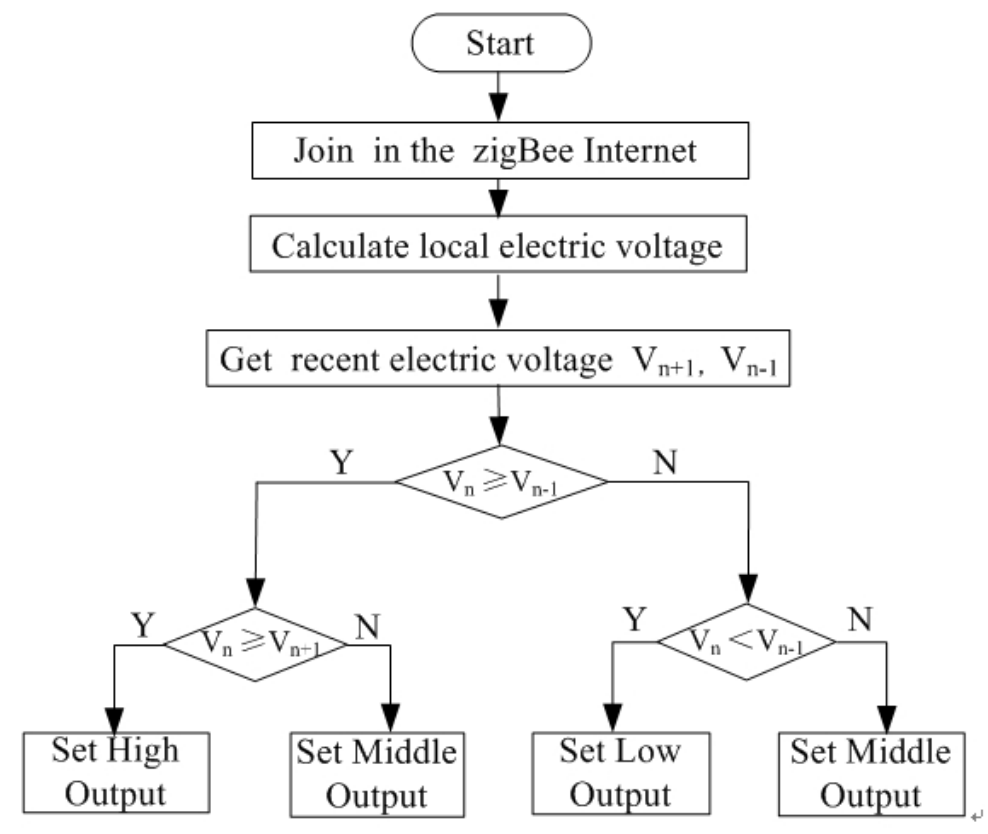

Fig.4. The Flow Chart of Output Coordination Strategy Algorithm

Compare the value of the battery energy storage between adjacent nodes. If the battery voltage of a node is higher than the battery voltage of the two adjacent nodes, we set its output current high and 
increase the illumination brightness. On the contrary, if the battery voltage is lower than the battery voltage of the two adjacent nodes, setting the output current is low and lower lighting levels. If the local voltage is centered, then set the output current is centered. This strategy can effectively protect the low storage nodes and reduce its output power, avoiding the situation that when it occurs consecutive rainy days, the individual lamps do not light up. Output coordination strategy can bring about comprehensive regulation of energy consumption of the entire system under the premise of guaranteed illumination, improving the reliability of independent solar power supply system.

\section{Conclusion}

Based on the extensive application of photovoltaic cells, this paper analyzes the problems existing in the independently solar-powered street lighting system and gives a program using the maximum power point tracking and output coordination strategy to improve the reliability. Application of the maximum power point tracking control method can improve the charging efficiency of the storage battery with photovoltaic cells. Using output coordination strategy can make each battery of the system work in coordination, effectively improving the continuous lighting time of the streetlight. The program has features of simple structure, convenient control, low cost, high efficiency, less precision sensors, etc.. Through testing, there are also very good tracking results even in the condition that the weather changes rapidly. And it can effectively improve the situation that independent solar-powered streetlights fail caused by the regional or sudden consecutive rainy weather.

\section{Acknowledgement}

In this paper, the research was sponsored by the Qinhuangdao city science and technology research and development plan program (Project No. 201401A012).

\section{References}

[1] Vasaki Ponnusamy, Low Tang Jung, Anang Hudaya. ZigBee Wireless Communication for Monitoring Renewable Street Light System, IEEE 2014: 978-1-4799-0059-6/13

[2] Z. L. Fabio Leccese, Intelligent wireless street lighting system, Venice, Environment and Electrical Engineering (EEEIC), 2012, pp. 958 - 961

[3] Oscar L., Maria Teresa Penella, A New MPPT Method for Low-Power Solar Energy Harvesting, IEEE Transactions on Industrial Electronics, vol. 57, no. 9, September 2010, pp. 3129-3138

[4] Radin, Boris,One solution of the light source based on LED with radio-control provided with Zigbeecompatible microcontroller CC2530,19th Telecommunications Forum, TELFOR 2011 Proceedings of Papers, 2011 , pp.932-935

[5] Dnyaneshwar S. Karanjka, Dr. S. Chatterji and Shimi S. L. NITTTR, Dr. Amod Kumar CSIO, An Improved Current Feedback Based Maximum Power Point Tracking Controller for Solar Photo-Voltaic System, IEEE 2013:978-1-4673-5149-213

[6] Liu, Dan, Yu, Hong, Smart control for wireless indoor lighting system based on ZigBee technology, Applied Material Science and Related Technologies, 2014, AdvancedMaterials Research, v 898, pp. 792-796

[7] B.Subudhi, R. Pradhan, A comparative study on maximum power point tracking t echniques for photovoltaic power systems, IEEE Transactions on Sustainable Energy, vol. 4, no. 1, January 2013, pp. 89-98 